\title{
Three-dimensional face-centered-cubic photonic crystal templates by laser holography: fabrication, optical characterization, and band-structure calculations
}

\author{
Yu. V. Miklyaev, D. C. Meisel, A. Blanco, and G. von Freymann ${ }^{\text {a) }}$ \\ Institut für Nanotechnologie, Forschungszentrum Karlsruhe in der Helmholtz-Gemeinschaft, \\ 76021 Karlsruhe, Germany \\ K. Busch \\ Institut für Theorie der Kondensierten Materie, Universität Karlsruhe (TH), 76128 Karlsruhe, Germany \\ W. Koch, C. Enkrich, M. Deubel, and M. Wegener \\ Institut für Angewandte Physik, Universität Karlsruhe (TH), 76128 Karlsruhe, Germany
}

(Received 9 September 2002; accepted 7 January 2003)

\begin{abstract}
We fabricate three-dimensional photoresist templates by means of laser holography. In particular, fcc structures are achieved by placing a specially designed "prism" onto the photoresist surface. This solves the problem of previous work, in which the refraction at the air-photoresist interface made it impossible to obtain the required angles of the light wave vectors inside the photoresist. The photoresist templates are characterized by scanning electron microscopy as well as by optical transmission spectroscopy, which agree well with numerical band-structure calculations. (C) 2003 American Institute of Physics. [DOI: 10.1063/1.1557328]
\end{abstract}

Large-area, three-dimensional (3D) photonic crystals ${ }^{1,2}$ incorporating cavities and waveguides would not only offer interesting perspectives in high-density integrated optics for telecommunications applications, but would also be interesting for fundamental studies, for instance on the quantum optical properties of nanoresonators. ${ }^{3,4}$ Different approaches towards this goal are currently being followed, such as inverse opals, ${ }^{5}$ stacked two-dimensional structures fabricated by electron-beam lithography, ${ }^{6,7}$ and holographically generated photoresist templates ${ }^{8-11}$ with the potential to be infiltrated by appropriate materials.

In the latter holographic approach, $N$ collimated coherent laser beams are sent onto a photoresist. The resulting multiple-beam interference pattern exposes the photoresist. In the developer, the more exposed and less exposed parts have different solubilities, leading to a porous photoresist structure. To obtain a 3D intensity pattern $I(\mathbf{r})$, which exposes the photoresist, $N$ needs to be four or larger [Fig. 1(a)]. The light intensity for $N=4$ can be written as

$$
I(\mathbf{r}) \propto\left|\sum_{n=1}^{4} \mathbf{E}_{n}^{0} \mathrm{e}^{\mathrm{i}\left(\mathbf{k}_{n} \cdot \mathbf{r}-\omega t\right)}\right|^{2}=\sum_{n, m=1}^{4} a_{n m} \mathrm{e}^{\mathrm{i} \mathbf{G}_{n m} \cdot \mathbf{r}} .
$$

Here, the reciprocal lattice vectors $\mathbf{G}_{n m}=\mathbf{k}_{n}-\mathbf{k}_{m}$ are determined by the differences of the wave vectors $\mathbf{k}_{n}$ of the incident plane waves and the (generally complex) form factors $a_{n m}=\mathbf{E}_{n}^{0} \cdot \mathbf{E}_{m}^{0^{*}}$, resulting from the relative amplitudes and polarizations of the incident laser beams. Three of the reciprocal lattice vectors $\mathbf{G}_{n m}$, for instance $\mathbf{G}_{12}, \mathbf{G}_{13}$, and $\mathbf{G}_{14}$, are linearly independent and form a basis of reciprocal space. The terms with $n=m$ lead to a constant background. Therefore, the reciprocal lattice vectors $\mathbf{G}_{n m}$ determine the lattice of the crystal structure, whereas the form factors $a_{n m}$ deter-

${ }^{\text {a)} E l e c t r o n i c ~ m a i l: ~ g e o r g . f r e y m a n n @ p h y s i k . u n i-k a r l s r u h e . d e ~}$ EVA-STAR (Elektronisches Volltextarchiv - Scientific Articles Repository) mine the internal structure of the unit cell. While Ref. 8 used linear polarizations only, choosing different linear, circular, or generally elliptical polarizations results in more design freedom via the form factors $a_{n m}$.

In order to achieve a complete 3D photonic band gap, it is known that symmetric structures are advantageous as compared to more asymmetric structures. ${ }^{12}$ An interesting candidate might be the fcc crystal structure (analogous to the inverse opals or diamond structures). In the pioneering work of Ref. 8, a bcc reciprocal lattice (equivalent to a fcc real-space lattice) was demonstrated in air.

However, it is important to note that this fcc lattice in air translates into a non-fcc, strongly asymmetric lattice [Figs. 1(b) and 1(c)] within the photoresist because of the refraction of the four beams at the air-photoresist interface. In particular, the realization of a fcc lattice in real space requires an angle of incidence for beams 2,3 , and 4 of $\gamma=\arccos (7 / 9)$ $=38.94^{\circ}$ with respect to the normal (beam 1), leading to the corresponding bcc lattice in reciprocal space. ${ }^{13}$ Even using grazing incidence, apart from being impractical, the critical angle of total internal reflection $\alpha_{\text {crit }}=\arcsin \left(1 / n_{\text {photoresist }}\right)$ generally does not allow one to obtain the required angle $\gamma$ inside the photoresist. For example, the photoresist used in Refs. 8 and 10 and in this article corresponds to a critical angle of total internal reflection of $36.78^{\circ}\left(n_{\text {photoresist }}\right.$ $=n_{\mathrm{SU}-8}=1.67$ for the undeveloped photoresist at $355-\mathrm{nm}$ wavelength).

In this letter, we extend the holographic technique by introducing a specially designed "prism" on top of the photoresist, which solves the refraction problem. This allows us to fabricate a fcc lattice in the photoresist with a lattice constant of about 550-nm. Analogous to the inverse opals, this periodicity would lead to a band gap in the visible or nearinfrared range for a sufficiently high refractive index contrast. The photoresist templates are characterized by scanning 84 
(a)
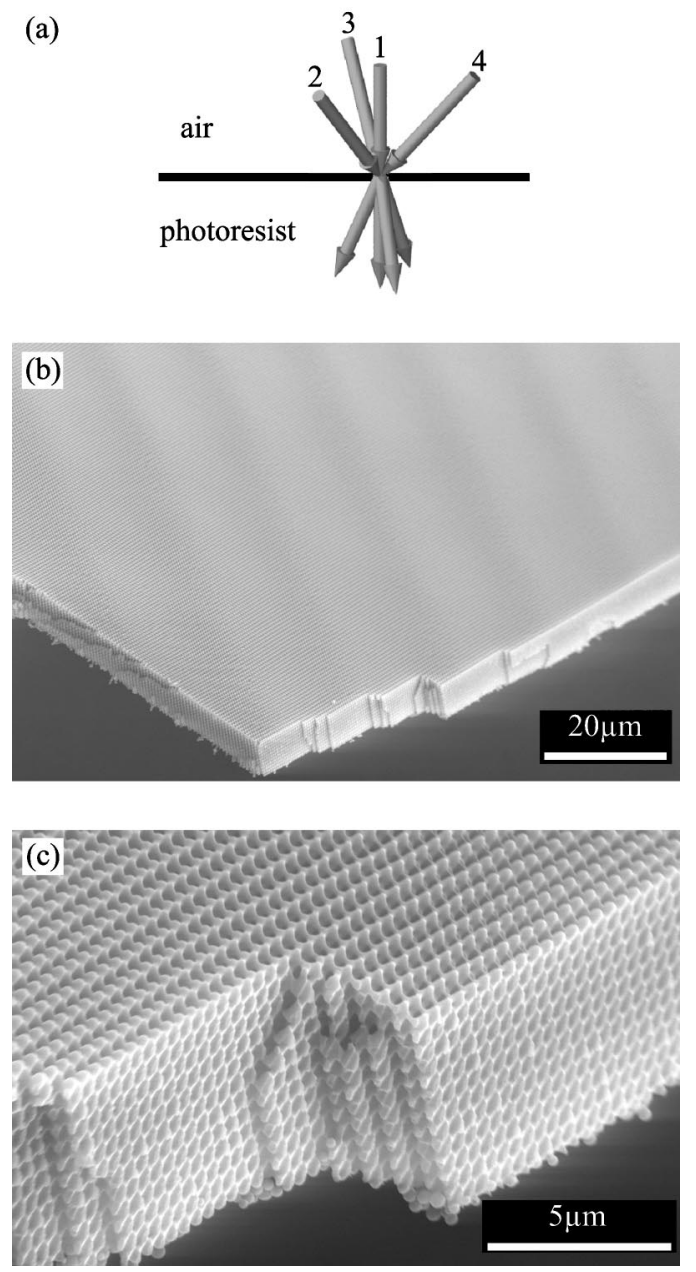

FIG. 1. (a) Scheme of laser holography employing four laser beams in the "umbrella-like" configuration, with the central beam, 1, and the three others, 2,3 , and 4 , symmetrically arranged with angle $\gamma=40.3^{\circ}$ with respect to the normal. Due to refraction at the air-photoresist interface, the angles inside the photoresist are smaller, leading to an asymmetric (i.e., trigonal) structure. (b) and (c) Corresponding experimental result. Electron micrographs of a broken edge of the developed photoresist template in two different magnifications.

electron microscopy as well as by optical transmission spectroscopy.

We use the thick-film photoresist SU-8 25 from MicroChem Corporation, which has a nominal resolution below $100 \mathrm{~nm}$. SU-8 does not exhibit a change of its refractive index during exposure, and has low absorption in the nearUV. The latter allows us to realize many lattice constants into the depth. For the exposure, we split the output of a frequency-tripled, Q-switched, single-mode Nd:YAG laser with about 6-ns pulse width into four beams, the intensity and polarization of each can be adjusted independently by means of half- and quarter-wave plates. The central beam, 1 , is circularly polarized, and the remaining beams are linearly polarized in their planes of incidence. The energy ratios (beam 1:2:3:4) are 1.5:1:1:1. The photoresist is exposed in just a single laser shot at $355-\mathrm{nm}$ wavelength with a total energy of about $8-\mathrm{mJ}$. The beam diameter is $8 \mathrm{~mm}$. After exposure, the photoresist is post-baked ${ }^{14}$ and developed.

The electron micrographs depicted in Figs. 1(b) and 1(c) show the result obtained through the exposure geometry shown in Fig. 1(a), leading to a highly asymmetric non-fcc structure, similar to the results reported in Ref. 8. In order to Downloaded 06 Mar 2009 to 129.13.72.198. Redistribution subject
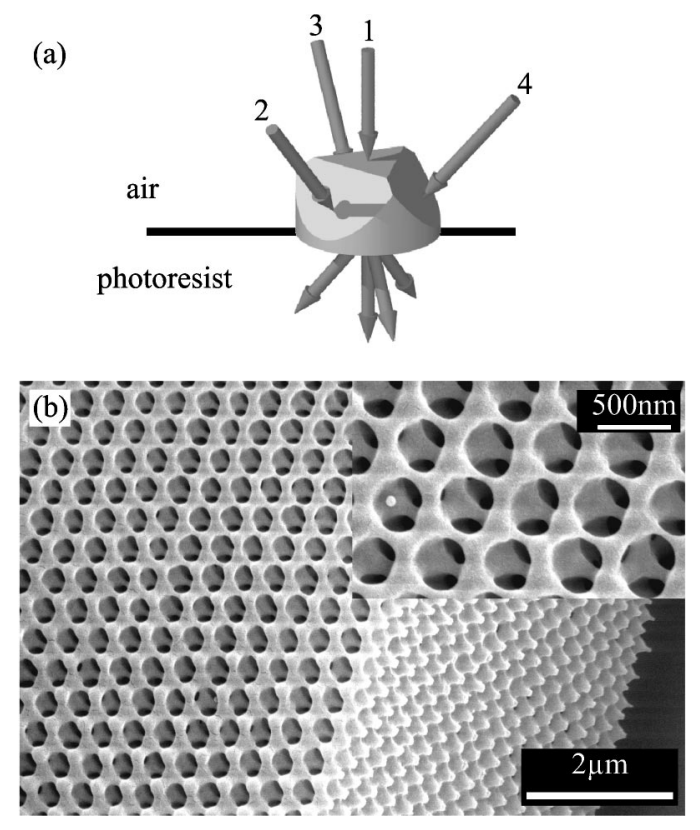

FIG. 2. (a) Scheme of laser holography with an additional corner cube with chopped-off tip on top of the photoresist. Here, the angles of the laser beams inside the photoresist correspond to a bcc reciprocal space lattice, equivalent to a real-space fcc lattice in the photoresist. (b) Corresponding experimental result. Electron micrograph of a broken edge of the developed photoresist template. The (111) direction is normal to the surface of the photoresist film.

fabricate a real-space fcc lattice, we place a specially designed, custom-made prism, that is, a corner cube with a chopped-off tip, onto the photoresist [Fig. 2(a)]. The light is coupled through the fused silica prism (refractive index $n$ $=1.48$ at $355-\mathrm{nm}$ wavelength), and through a thin layer of distilled water into the photoresist $\left(n_{\mathrm{SU}-8}=1.67\right.$ for the undeveloped photoresist at $355-\mathrm{nm}$ wavelength). From these parameters, we calculate a fcc structure with a nominal lattice constant of $a=550 \mathrm{~nm}$. The resulting photoresist structure shown in Fig. 2(b) is indeed close to fcc.

We characterize these fcc templates by transmission optical spectroscopy along the (111) direction with a spot size of about $100 \mu \mathrm{m}$. The intensity transmission spectrum [righthand side (RHS) in Fig. 3] exhibits a pronounced dip around $\lambda_{0}=680-\mathrm{nm}$ wavelength. The transmission in this minimum is $13 \%$. In order to compare the optical transmission experiment with rough estimates or with band-structure calcula-

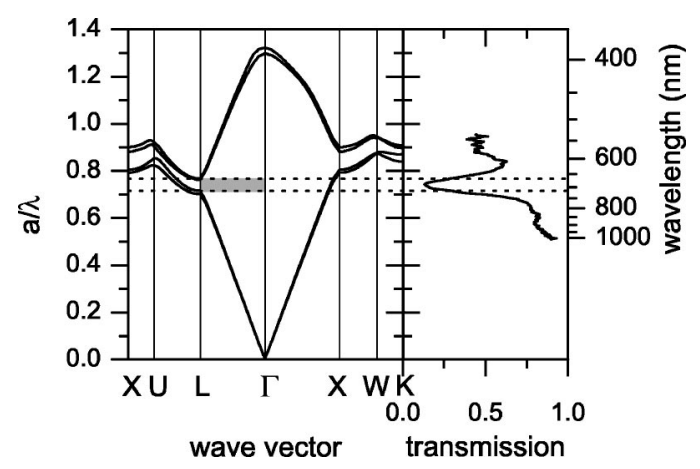

FIG. 3. Optical intensity transmission spectrum (RHS) of the fcc photoresist template in Fig. 2(b) along the (111) direction $(\Gamma L)$, showing a minimum in transmission around 680-nm wavelength. The left-hand side exhibits a corresponding band-structure calculation. The gray shaded area corresponds to the direction measured. The dotted lines are guides for the eye. 
tions, one needs to know the index of refraction of the developed photoresist in the relevant wavelength region. We obtain an index of $n_{\mathrm{SU}-8}=1.61$ at $633-\mathrm{nm}$ wavelength from an independent measurement of Brewster's angle on a developed bulk photoresist layer. From an inspection of the images shown in Fig. 2(b) and from calculations of the structure, we estimate a photoresist filling fraction of $f=29 \%$. Furthermore, it is known that the thickness of SU-8 films shrinks during development. For instance, Ref. 15 reports a shrinkage of $7.5 \%$, leading to a lattice constant along (111) of $a=509 \mathrm{~nm}$. To get a rough estimate of the center wavelength $\lambda_{0}$ of the fundamental stop band, we employ Bragg's law

$$
\lambda_{0}=2 d_{111} n_{\mathrm{eff}},
$$

with the distance between adjacent lattice planes in the (111) direction, $d_{111}=294 \mathrm{~nm}$, and the effective refractive index of the photoresist template $n_{\text {eff }}=\sqrt{n_{\text {SU- }}^{2} f+(1-f)}$. This leads to $\lambda_{0}=711 \mathrm{~nm}$, consistent with the experiment [Fig. 3 (RHS)].

In order to compute not only the center wavelength of the stop band but also its width, we have performed bandstructure calculations for the structure depicted in Fig. 2(b) using a plane-wave expansion method ${ }^{12,16}$ with the parameters given earlier. The results of this calculation for the measured (111) direction $(\Gamma L)$ are presented in Fig. 3. The remaining slight anisotropy of the internal structure of the unit cell with respect to the fcc symmetry group leads to a splitting of the two lowest bands. As a consequence, the irreducible Brillouin zone (IBZ) associated with the structure of Fig. 2(b) is larger than the standard IBZ for isotropic fcc structures, and care has to be exerted when comparing with the measured transmission data. The calculated energetic position as well as the spectral width of the corresponding stop gap agree well with the experiment (Fig. 3).

In conclusion, we have fabricated a fcc structure in a photoresist by means of laser holography. This has become possible by introducing a specially designed corner cube with chopped-off tip on top of the photoresist, which overcomes the fundamental problem that, without such prism, the required angles in the photoresist cannot be achieved due to refraction of light at the air-photoresist interface. (Previous work demonstrated fcc lattices in air which corresponded to highly asymmetric non-fcc, i.e., trigonal structures of the photoresist itself.) Moreover, we have introduced improved design freedom of the internal structure of the unit cell by employing different polarizations of the four incident laser beams by, for example, circular polarization of the central beam and linear polarizations of the other three beams. This design freedom leads to a more symmetric internal structure of the unit cell, which is desirable for achieving complete 3D photonic band gaps after infiltration of the templates with a high-index material. The photoresist templates are characterized by optical transmission spectroscopy. These measurements agree well with corresponding band-structure calculations.

We acknowledge support by the Deutsche Forschungsgemeinschaft (DFG) through the DFG-Forschungszentrum "Functional Nanostructures" (DFG-CFN). The research of one of the authors (K.B.) is further supported by DFGproject $\mathrm{Bu}$ 1107/2-1 (Emmy-Noether program), that of another author (M.W.) by the DFG Leibniz-award 2000.

${ }^{1}$ S. John, Phys. Rev. Lett. 58, 2486 (1987).

${ }^{2}$ E. Yablonovitch, Phys. Rev. Lett. 58, 2059 (1987).

${ }^{3}$ Photonic Crystals and Light Localization in the 21st Century, NATO Science Series C Vol. 563, edited by S. M. Soukoulis (Kluwer Academic, Dordrecht, 2001).

${ }^{4}$ J. D. Joannopoulos, R. D. Meade, and J. N. Winn, Photonic Crystals (Princeton University Press, Princeton, NJ, 1995).

${ }^{5}$ A. Blanco, E. Chomski, S. Grabtchak, M. Ibisate, S. John, S. W. Leonard, C. Lopez, F. Meseguer, H. Miguez, J. P. Mondia, G. A. Ozin, O. Toader, and H. M. van Driel, Nature (London) 405, 437 (2000).

${ }^{6}$ N. Yamomoto, S. Noda, and A. Chutinan, Jpn. J. Appl. Phys. 37, 1052 (1998); S. Noda, K. Tomoda, N. Yamomoto, and A. Chutinan, Science 289, 604 (2000)

${ }^{7}$ J. G. Flemming and S.-Y. Liu, Opt. Lett. 24, 49 (1999).

${ }^{8}$ M. Campbell, D. N. Sharp, M. T. Harrison, R. G. Denning, and A. J. Turberfield, Nature (London) 404, 53 (2000).

${ }^{9}$ S. Shoji and S. Kawata, Appl. Phys. Lett. 76, 2668 (2000).

${ }^{10} \mathrm{~S}$. Yang, M. Megens, J. Aizenberg, P. Wiltzius, P. M. Chaikin, and W. B. Russel, Chem. Mater. 14, 2831 (2002).

${ }^{11}$ V. P. Tondiglia, L. V. Natarajan, R. L. Sutherland, D. Tomlin, and T. J. Bunning, Adv. Mater. 14, 187 (2002).

${ }^{12}$ K. Busch and S. John, Phys. Rev. E 58, 3896 (1998).

${ }^{13}$ K. I. Petsas, A. B. Coates, and G. Grynberg, Phys. Rev. A 50, 5173 (1994).

${ }^{14}$ The samples are baked in a convection oven for 6 minutes while the temperature rises from 65 to $93{ }^{\circ} \mathrm{C}$.

${ }^{15}$ L. J. Guerin, M. Bossel, M. Demierre, S. Calmes, and P. Renaud, Proceedings of the International Solid State Sensors and Actuators Conference (Transducers '97), 1997, Vol. 2, p. 1419.

${ }^{16}$ K. M. Ho, C. T. Chan, and S. M. Soukoulis, Phys. Rev. Lett. 65, 3152 (1990). 